\title{
Extended galactic rotational velocity profiles in $f(R)$ gravity background
}

\author{
Vipin Kumar Sharma ${ }^{\mathrm{a}}$, Bal Krishna Yadav ${ }^{\mathrm{b}}$, Murli Manohar Verma ${ }^{\mathrm{c}}$ \\ Department of Physics, University of Lucknow, Lucknow 226 007, India
}

Received: 31 December 2019 / Accepted: 28 June 2020 / Published online: 13 July 2020

(C) The Author(s) 2020

\begin{abstract}
An attempt has been made to explore the geometric effects of $f(R)$ action on the galactic dynamics under the weak field approximation. The rotational velocity is calculated beyond the Einstein's geometric theory of gravity. It is inspired by the cosmological geometric relation obtained in the power-law $f(R)$ gravity model in vacuum. We analyse the action with a small positive deviation from the EinsteinHilbert gravity action (taking $R$ as $f(R) \propto R^{1+\delta}$ ) at the galactic scales for the explanation of the flatness paradox associated with the clustered galactic dark matter. We obtain the contribution of a dynamical $f(R)$ cosmological background geometry on accelerating the test mass. Furthermore, the integrated effective acceleration of the test mass due to a massive spherically symmetric source in $f(R)$ background is calculated via the study of geodesics for the suitable spacetime metric and an equation for the effective rotational velocity has been developed. We test the viability of the proposed model by tracing the motion of a test mass far from the disk of galactic matter for smaller $\delta$. The possible galactic rotational velocity curves in $f(R)$ background are discussed for the formula obtained with $\delta<<1$. We also obtain constraints on $\delta O\left(10^{-6}\right)$ confirmed by observations.
\end{abstract}

\section{Introduction}

Theoretical modelling of the observed strange behaviour of the galactic rotation curves [3-6], the gravitational lensing phenomenon (in the case of special cluster (1E 0657-558) $[7,8]$, the Supernovae Ia observation [9-11], etc., in the standard of theory of gravity, general relativity (GR), motivates one to include some dark components of about $95 \%$ of the total energy content of the universe. This dark sector fits well in the theoretical cosmological models in the GR framework

\footnotetext{
a e-mail: vipinastrophysics@gmail.com

b e-mail: balkrishnalko@gmail.com

c e-mail: sunilmmv@yahoo.com (corresponding author)
}

and explains the current observations of cosmology at different redshifts $(z)$, i.e., at galactic and extragalactic scales. On this basis, it reveals itself in two different features: (i) dark matter $(\approx 27 \%$ ) with zero pressure which is further categorized into baryonic (like Massive Astronomical Compact Halo Objects-MACHOs) or non-baryonic (like non-standard elementary particles-axions), and (ii) dark energy $(\approx 68 \%$ ) with negative pressure, following the Planck data 2018 [12]. Basically, the observable signature of the clustered dark matter is also associated with low redshifts, and hence scientists have been engaged to trace its presence in different groundand space-based projects. On the other hand, unclustered dark energy is mainly associated with a high redshift.

However, there is a big question mark in front of these dark components and all possible efforts to remove this are still under progress. Thus, this has become the major challenge in the current field of cosmology. Observational cosmology requires the presence of such dark components, but experimentally we are not able to pinpoint them through different projects, viz., LHC, IceCube, XENON-100, DAMA/LIBRA, CDMS II, Large Synoptic Survey Telescope, DES, and the proposed Dark Energy Space Telescope or Destiny.

Alternatively, as per the Einstein's geometric tensorial equations, one can make an attempt to specifically obtain the geometry of spacetime corresponding to the observations at different redshifts $(z)$ via modifying the Einstein-Hilbert gravity action. Generally, the literature describing the gravity theory beyond Einstein is vast and one can recast the modified gravity action into scalar-tensor, tensor-vector-scalar, higher order gravity theory, etc. [13-20]. Initial reproductions of the observed rotation velocity profile without dark matter include the Milgrom's modification of Newtonian dynamics [21-23]. Also, one of the simplest modifications of the standard Einstein's general relativistic (GR) dynamics is obtained just by taking $R$ as $f(R)$ and hence we have an extra degree of freedom called the scalaron (or scalar field). Initially, the study of the gravity theory via higher 
order Lagrangians was introduced by Buchdahl [24] and was subsequently carried out by Starobinsky for the study of cosmic evolution [25-27]. Thereafter, serious efforts have been made in this field for the theoretical explanation of the major observational issues via $f(R)$ cosmology [28-35].

Some important characteristic features of the $f(R)$ gravity regarding the screening mechanism and Noether symmetry (in particular, the emergence of a scaling length in a powerlaw $f(R)$ model), useful for our work, are discussed in [3641], although the field of $f(R)$ cosmology is very vast. Such features may lead one to mimic dual behaviour for the explanation of dark energy and dark matter for a unique power-law $f(R)$ model or scalar field model [42-44]. Some important implications of $f(R)$ or scalar field cosmology have been discussed in [45-48].

Apart from this, motivated by the Schwarzschild-like solution of the Einstein equations with a cosmological constant, i.e., Schwarzschild-de Sitter spacetime metric, one can also argue in an alternative way that the modification in the theory of gravity can influence the local geodesics and may explain the observational consequences (such as the flatness profile of rotation curves) on the galactic dynamics as well. We attempt to address this issue within $f(R)$ framework. Several important researches done so far in GR framework, signify the effects on the local dynamics due to the present status of the cosmological background. In precision cosmology, one can choose the quintessence, phantom or scalar fields to investigate such possibility instead of making the straight choice for the cosmological constant $(\Lambda)$ term [49-54]. Here, we want to emphasize a remarkable feature of the Schwarzschild spacetime metric, i.e., the Einstein equations become linear for such a spacetime metric and hence it is possible to combine or superpose different solutions.

In principle, the study of the effects of expansion (or acceleration) of the background universe on the local system can be traced back to the gravitational action of the background universe which is in favour of Mach's principle in cosmology. Here, we study such effects on the galactic dynamics for the explanation of clustered galactic dark matter problem in the power-law $f(R)$ model via a suitable spacetime metric element under the weak field limits for the test mass (hydrogen atom). It is also an important task in cosmology to obtain an unique spacetime metric element which can clearly interpolate between local (Schwarzschild static metric) and global scales (non-static FLRW (Friedmann-Lemaitre-RobertsonWalker) metric) and hence may join the two solutions.

Thus, we work with the specific $f(R)$ model which has a small positive deviation parameterized by $\delta$ from the standard Einstein-Hilbert action for the explanation of galactic dark matter by taking $R$ as $f(R)=\frac{R^{1+\delta}}{R_{C}^{\delta}} ; R_{C}$ is the weight constant in $f(R)$ background having the dimension of the Ricci scalar. Hence, the key idea in the present paper is to modify the spacetime geometry according to the form of the power-law $f(R)$ gravity model and study its effects on the galactic dynamics by exploring the spacetime metric element in the weak gravitational field limit for the explanation of clustered galactic dark matter problem. We, therefore, obtain the solution of the $f(R)$ model in terms of the scale factor $a(t)$ and then calculate an integrated effective acceleration equation in the presence of a massive spherically-symmetric source of mass $M$ for a test mass in $f(R)$ background via the study of a suitable spacetime metric in the Newtonian limit. Next, we develop an equation for the rotational velocity in the dynamical $f(R)$ background i.e., $f(R) \neq R$ for the bounded system.

Accordingly, our work is organized as follows. In Sect. 2, the dynamics of $f(R)$ model inspired by the Ricci scalar curvature $(R)$ is discussed. In Sect. 3, the solution and the potential of the $f(R)$ background is obtained. In Sect. 4 , we discuss the local geodesics in $f(R)$ background via the suitable spacetime metric element in the weak gravitational field limit and calculate the effective $f(R)$ rotational velocity equation and further investigate its behaviour for the model parameter $\delta$. We discuss the galactic dynamics for the possible fits beyond the luminous disk of galaxy matter by exploring the galactic scaling parameter $r_{0}$ in the effective $f(R)$ rotational velocity equation for the typical galactic data with $\delta<<1$ in Sect. 5. We conclude and discuss our work in Sect. 6.

Throughout the paper, we use the signature of the spacetime metric $(-,+,+,+)$, the indices $\mu, v=0,1,2,3$ and $\mathrm{c}=\hbar=1$.

\section{Curvature scalar inspired $f(R)$ dynamics}

The main motivation to work with the fourth order gravity, i.e., $f(R)$ is that it may fill the gap of the dark sectors without actually requiring them to be in the strange or exotic form under the suitable stability conditions of the $f(R)$ model. Here, we assume that the background universe is homogeneous, isotropic and spatially flat. This is given by the Friedmann-Lemaitre-Robertson-Walker (FLRW) spacetime metric as

$\mathrm{d} s^{2}=-\mathrm{d} t^{2}+a^{2}(t)\left[\mathrm{d} r^{2}+r^{2}\left(\mathrm{~d} \theta^{2}+\sin ^{2} \theta \mathrm{d} \phi^{2}\right)\right]$,

where $a(t)$ is the time dependent cosmological scale factor and $(r, \theta, \phi)$ are the usual spherical polar coordinates. The $f(R)$ dynamics in such a spacetime is governed by the 4dimensional modified gravity action with the standard matter,

$\mathcal{A}=\int d^{4} x \sqrt{-g}\left[\frac{1}{16 \pi G} f(R)+L_{m}\left(g_{\mu \nu}, \Psi_{m}\right)\right]$, 
where $L_{\mathrm{m}}$ is the Lagrangian of the standard matter component with the matter field $\Psi_{m}, g$ is the determinant of the metric tensor $g_{\mu \nu}$ and $G$ is the Newtonian gravitational constant. In the metric formalism, the modified gravity field equations are obtained by varying the action integral (2) w.r.t. $g_{\mu \nu}$,

$$
\begin{array}{r}
F(R) R_{\mu \nu}-\frac{f(R) g_{\mu \nu}}{2}-\nabla_{\mu} \nabla_{\nu} F(R) \\
+g_{\mu \nu} \square F(R)=8 \pi G T_{\mu \nu}^{(m)},
\end{array}
$$

where $F(R)$ is the first derivative of $f(R)$ w.r.t. the Ricci scalar $R, R_{\mu \nu}$ is the Ricci curvature tensor, $\square\left(\equiv \nabla^{\mu} \nabla_{\mu}\right)$ is the covariant Laplacian, $T_{\mu \nu}^{(m)}$ is the energy-momentum tensor of matter and $\nabla_{\mu}$ is the covariant derivative associated with the Levi-Civita connection of the metric. We can also rewrite Eq. (3) in the standard form of modified Einstein tensor equations,

$G_{\mu \nu}=\frac{8 \pi G}{F(R)}\left[T_{\mu \nu}^{(c)}+T_{\mu \nu}^{(m)}\right]$,

where

$$
\begin{aligned}
T_{\mu \nu}^{(c)}= & \frac{1}{8 \pi G}\left[\frac{1}{2} g_{\mu \nu} f(R)-\frac{R}{2} g_{\mu \nu} F(R)\right. \\
& \left.+\nabla_{\mu} \nabla_{\nu} F(R)-g_{\mu \nu} \square F(R)\right]
\end{aligned}
$$

is the energy-momentum tensor of the spacetime curvature. Here, it is to be noted that the Ricci scalar is dynamical if $f(R) \neq R$, otherwise the theory gets reduced to the standard GR. Also, the constant function, $f(R)$, added or subtracted from the Ricci scalar of the Einstein-Hilbert gravity action acts like the Einstein's cosmological constant.

Thus, the dynamical form of $f(R)$ introduces a new degree of freedom, called the scalaron, which can explain various cosmological phenomena at different redshifts. Also, the extra curvature term which appears in Eq. (4), apart from the usual Einstein tensor $\left(G_{\mu \nu}\right)$ in the vacuum case, will determine the existence of dark matter or dark energy.

Now, from Eq. (1), we can obtain an equation for the Ricci scalar:

$R=6\left[2 H^{2}+\dot{H}\right]$,

where $H$ is the Hubble expansion parameter and the overdot represents the cosmic time derivative. Also, from the field equations of $f(R)$ for the vacuum case we find

$3 F(R) H^{2}=\frac{1}{2}[F(R) R-f(R)]-3 H \dot{F}(R)$.

Here, we address the problem of clustered dark matter at the galactic scales by considering a mild modification of gravity of the form

$f(R)=\frac{R^{1+\delta}}{R_{c}^{\delta}}$, where $R_{\mathrm{c}}$ is the weight constant and has the dimension of the Ricci scalar, while $\delta$ is a dimensionless quantity which we assume to be small. Such a model can explain the signature of clustered dark matter at low redshifts in the rotational velocity profiles of $H_{\alpha}$ and $\mathrm{HI}$ outside the typical galaxies in the $f(R)$ background [39-41,55-57].

We can simply investigate the role of the power-law $f(R)$ model for the parameter $\delta$ that signifies the deviation from GR for the explanation of the background evolution. For the small deviation parameterized by $\delta$, we can perform a Taylor expansion of Eq. (8) as

$f(R) \equiv R^{1+\delta} \approx R+\delta g(R)+O(2) \ldots$

For $\delta<<1$, we have from Eq. (9)

$f(R) \approx R+\delta g(R)$.

Equation (10) looks similar to the Einstein-Hilbert gravity action with the cosmological constant term $(\Lambda)$, only if the second term is constant. The variation of Eq. (10) w.r.t. $R$ thus suggests the role of the power-law model in determining the dynamical background evolution, since $f(R) \neq R$.

We now proceed to obtain the background geometry of the spacetime for such a model by solving the modified gravity field equations for the vacuum case.

Making use of Eq. (6), we express the time derivative $\left(\frac{\mathrm{d}}{\mathrm{d} t}\right)$ as

$\frac{\mathrm{d}}{\mathrm{d} t} \equiv \frac{\mathrm{d} H}{\mathrm{~d} t} \frac{\mathrm{d}}{\mathrm{d} H}=\dot{H} \frac{\mathrm{d}}{\mathrm{d} H}=\left(\frac{R}{6}-2 H^{2}\right) \frac{\mathrm{d}}{\mathrm{d} H}$.

Next, we use Eqs. (8) and (11) in Eq. (7) and obtain

$\frac{\left(R-12 H^{2}\right)}{(\delta-1)}\left[H \frac{\mathrm{d} R}{\mathrm{~d} H} \delta(1+\delta)-R \delta\right]=6 R H^{2}$.

On using Eq. (6) in Eq. (12), we get

$$
\begin{aligned}
& 6 \delta \dot{H}\left[H \frac{\mathrm{d} R}{\mathrm{~d} H}(1+\delta)-R\right]-\frac{R^{2}(\delta-1)}{2} \\
& +3 R \dot{H}(\delta-1)=0,
\end{aligned}
$$

which can be solved further on dividing Eq. (13) throughout by RH to give

$$
\begin{aligned}
& 6\left[\frac{\mathrm{d} R}{R \mathrm{~d} t}\right] \delta(1+\delta)-3 \delta\left[\frac{\mathrm{d} H}{H \mathrm{~d} t}\right]-3\left[\frac{\mathrm{d} H}{H \mathrm{~d} t}\right] \\
& -\left[\frac{R}{2 H}\right](\delta-1)=0 .
\end{aligned}
$$

Now, using Eq. (6) in the last term of Eq. (14), we get

$6\left[\frac{\mathrm{d} R}{R \mathrm{~d} t}\right] \delta(1+\delta)-6 \delta\left[\frac{\mathrm{d} H}{H \mathrm{~d} t}\right]+6 H(1-\delta)=0$,

which can be solved to give

$\left[\frac{R}{R_{0}}\right]^{\delta(1+\delta)}=\left[\frac{\dot{a}(t)}{\dot{a}\left(t_{0}\right)}\right]^{\delta}(1+z)$. 
In this approach, the cosmological evolution naturally relates to the behaviour of $f(R)$ spacetime geometry at very low cosmological redshifts for the explanation of clustered galactic dark matter whose dynamics can be expressed as

$R \approx R_{0}\left[\frac{H}{H_{0}}\right]^{\frac{1}{1+\delta}}$.

This solution sets the relation between $R$ and $H$ with $R_{0}$ and $H_{0}$ as the constants representing the present values of the Ricci scalar curvature and the Hubble expansion parameter, respectively. Using such a cosmological solution, it is possible to study its effect on the local galactic dynamics. This provides us with strong motivation to draw the cosmological dynamics close to the galactic environment as dealt with next. In the further sections, we do this via joining the cosmological background solution with the local solution.

\section{Calculation of potential for the $f(R)$ background}

We proceed with Eq. (11) and explore the behaviour (or solution) of the scale factor $a(t)$ for the proposed geometric form of $f(R)$ model. Therefore, from Eq. (17), we get

$\int \frac{\mathrm{d} a}{a(t)}=\int \frac{H \mathrm{~d} H}{\frac{R}{6}-2 H^{2}}$,

which gives

$a(t)=a_{0}\left[\frac{1-\chi}{\left(\frac{H}{H_{0}}\right)^{\frac{1+2 \delta}{1+\delta}}-\chi}\right]^{\frac{1+\delta}{2+4 \delta}}$,

where $\chi=\frac{R_{0}}{12 H_{0}^{2}}$ and $a_{0}$ is the present value of the scale factor. Now, Eq. (19) can be recast as

$H=\frac{\dot{a}}{a}=H_{0}\left[\chi-(\chi-1)\left(\frac{a}{a_{0}}\right)^{-\left(\frac{2+4 \delta}{1+\delta}\right)}\right]^{\frac{1+\delta}{1+2 \delta}}$.

We can easily obtain the contribution of the dynamical $f(R)$ cosmological background geometry in the case of an accelerating test mass:

$$
\begin{aligned}
\ddot{a}= & \frac{H_{0}^{2}}{a^{3}}\left[\chi a^{\frac{2+4 \delta}{1+\delta}}-(\chi-1) a_{0}{ }^{\frac{2+4 \delta}{1+\delta}}\right]^{\frac{1}{1+2 \delta}} \\
& \times\left[\chi a^{\frac{2+4 \delta}{1+\delta}}+(\chi-1) a_{0}{ }^{\frac{2+4 \delta}{1+\delta}}\right] .
\end{aligned}
$$

Since $a_{0}>a$ always, so for the $f(R)$ background geometry, the acceleration of the test mass in $f(R)=\frac{R^{(1+\delta)}}{R_{c}^{\delta}}$ type models is negative for $\chi \approx \frac{1}{2}$ (obtained by using the current standard model of cosmology, i.e., the $\Lambda$-CDM model, which estimates the value of $R_{0} \approx 6 H_{0}^{2}$ ), i.e., the test mass is decelerated in this $f(R)$ background geometry, which is a feature favourable for the explanation of the observable signature of clustered galactic dark matter on the galactic dynamics. Hence, the potential of the $f(R)$ background geometry for the test mass will be given by using the basic kinematic definition

$V_{f(R)}=-\int \ddot{a} \mathrm{~d} a$.

Thus, we have from Eqs. (21) and (22) the spacetime background $f(R)$ potential,

$V_{f(R)}=-\frac{H_{0}^{2}}{2 a^{2}}\left[\chi a^{\frac{2+4 \delta}{1+\delta}}-(\chi-1) a_{0}^{\frac{2+4 \delta}{1+\delta}}\right]^{\frac{2(1+\delta)}{1+2 \delta}}$,

where all symbols have the usual meaning as mentioned earlier.

It is interesting to determine the effects of this background $f(R)$ potential on the local dynamics and also to obtain the rotational velocity profile for the test mass [54-56].

\section{Local geodesics and rotational velocity in $f(R)$ background}

The spherically symmetric Schwarzschild-like spacetime metric has the unique feature that it can reduce the Einstein field equations to the linear form so that we can superpose different solutions and the Schwarzschild-de Sitter metric emerges as one of its important consequences. The motivation to investigate the effects of background evolution on the local dynamics comes from this Schwarzschild-de Sitter spacetime metric which involves the cosmological constant $(\Lambda)$ along with the Newtonian term and hence opens the possibility to investigate the equations of motion. Alternatively, instead of using the cosmological constant term $(\Lambda)$, one can choose the quintessence, phantom or scalar fields to explore such a possibility [49-54]. The modification of the gravity action generally introduces extra degrees of freedom in the form of a scalar field. Therefore, in order to analytically discuss the local geodesics in the $f(R)$ background, it is crucial to choose the specific spacetime metric element from which the geodesic equations can be determined, and hence one may consider joining different solutions.

Several serious efforts have been made so far in order to generalize the spacetime metric element which can interpolate between local and global scales [51,52,54]. Baker has discussed different approaches regarding this problem $[51,52]$. However, we still do not have the detailed and unique form of such an interpolating spacetime metric. Hence, with the solutions motivated by spherical symmetry, we can generalize the Schwarzschild spacetime metric element outside the spherically symmetric source in comoving and isotropic coordinates in the weak gravitational field limit $[54,58]$, 


$$
\begin{aligned}
\mathrm{d} s^{2} \approx & -\left(1-\frac{2 G M}{a(t) \rho}\right) \mathrm{d} t^{2}+a(t)^{2} \\
& \times\left[\mathrm{d} \rho^{2}+\rho^{2}\left(\mathrm{~d} \theta^{2}+\sin ^{2} \theta \mathrm{d} \phi^{2}\right)\right],
\end{aligned}
$$

where $\rho$ is the comoving radial coordinate such that $\rho=\frac{r}{a(t)}$.

Because of the spherical symmetry of the Schwarzschild metric element, we confine our attention to the test mass motion in the equatorial plane $\left(\theta=\frac{\pi}{2}\right)$. Therefore, the effective radial equation of motion for a test mass in the weak gravitational field limit is

$\ddot{r}_{e f f}=\frac{\ddot{a}}{a} r-\frac{G M}{r^{2}}$.

An alternative way to obtain Eq. (25) in the weak field limit is discussed in [51,52,59].

The solution of modified gravity is obtained in terms of the scale factor in Sect. 3. Therefore, we can join the two solutions.

Now, we study the implication of this equation in the $f(R)$ background via developing the rotational velocity equation for the bounded system. In order to support the study of the similar effects of the clustered galactic dark matter signature on the rotational velocity profile via the spectroscopic measurement of hydrogen atom (test mass) at local scale, we study the nearby bounded system in the proposed $f(R)$ spacetime geometry background. Thus, it is possible to rewrite Eq. (23) using the Planck system $(\mathrm{c}=\hbar=1)$ of units (such that $\frac{a_{0}}{a}=\frac{r_{0}}{r}$ ), as

$V_{f(R)}=-\frac{H_{0}^{2}}{2 r^{2}}\left[\chi r^{\frac{2+4 \delta}{1+\delta}}-(\chi-1) r_{0} \frac{2+4 \delta}{1+\delta}\right]^{\frac{2(1+\delta)}{1+2 \delta}}$.

Here, we regard $r_{0}$ as the $f(R)$ background free length scale parameter at the galactic scales according to [39-41], which is a fundamental feature of power-law $f(R)$ gravity. Therefore, it may be the size of spherical galactic halo of scalaron clouds apart from the dark matter halo (measured in $\mathrm{kpc}$ ), needed for the explanation of the flatness profile in the outer region of galaxy.

Thus, for the bounded motion of the test mass under the influence of gravitating body in the $f(R)$ background, we can develop the rotational velocity by using Eq. (25) and the basic kinematic definition

$v \simeq \sqrt{\left|\ddot{r}_{\text {eff }} r\right|}$.

We have the rotational velocity of the test mass in $f(R)$ background geometry

$$
\begin{aligned}
v^{2} & \simeq \frac{G M}{r}+(\chi)^{\left(\frac{2+2 \delta}{1+2 \delta}\right)} H_{0}{ }^{2} r^{2}\left[1+\frac{(\chi-1)}{\chi}\left(\frac{r_{0}}{r}\right)^{2\left(\frac{1+2 \delta}{1+\delta}\right)}\right] \\
& \times\left[1-\frac{(\chi-1)}{\chi}\left(\frac{r_{0}}{r}\right)^{2\left(\frac{1+2 \delta}{1+\delta}\right)}\right]^{\frac{1}{1+2 \delta}}
\end{aligned}
$$

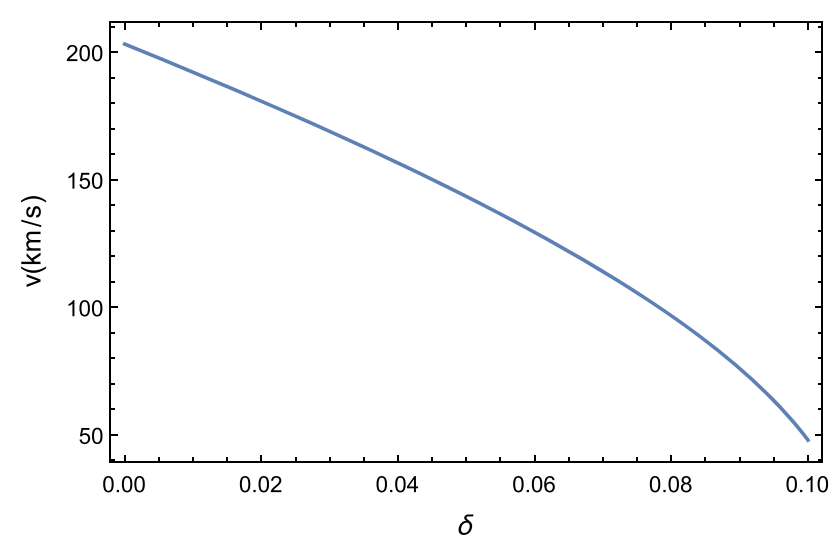

Fig. 1 The theoretical effective $f(R)$ rotation velocity curve for a typical massive galaxy shows the variation w.r.t. the parameter $\delta<1$ with $\frac{r_{0}}{r}>1$

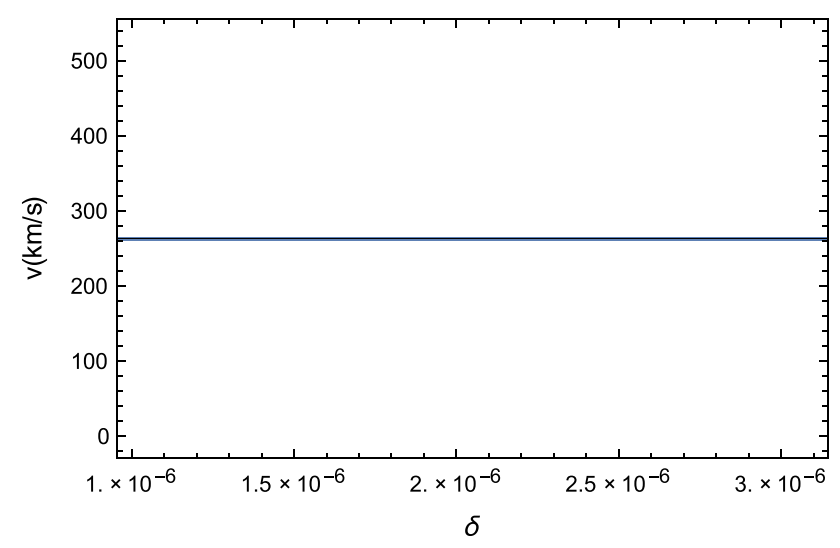

Fig. 2 The theoretical effective $f(R)$ rotation velocity curve w.r.t. the model parameter $\delta<<1$ with $\frac{r_{0}}{r}>1$. It shows that the rotational velocity attains a constant value for different values of the parameter $\delta$. The smaller value of $\delta$ seems to be consistent with the explanation of the effects of dark matter on the rotational velocity of the test mass in the outer regions of the galaxy

where the current value of $H_{0}$ (from the Planck data), $H_{0}=$ $67.4 \pm 0.5 \mathrm{~km} \mathrm{~s}^{-1} \mathrm{Mpc}^{-1}$ [12], the value of the Newtonian gravitational constant, $G=4.3 \times 10^{-6} \mathrm{kpc} \mathrm{km}^{2} \mathrm{~s}^{-2} M \stackrel{-1}{\odot}$ and $\chi\left(=\frac{R_{0}}{12 H_{0}^{2}}\right) \approx \frac{1}{2}$.

The first term of Eq. (28) predicts the Keplerian curve for the constant mass (hence a point source), whereas the second term is due to the $f(R)$ background contribution. As we move away from the mass concentration $M$, the anomaly in the rotational velocity can be explained without any particulate dark matter but through the mild modification of Einstein-Hilbert gravity. The behaviour of the effective $f(R)$ rotational velocity curve for different values of $\delta$ is plotted in Figs. 1 and 2 .

Figure 1 shows that $f(R)$ rotational velocity decreases for slightly higher values of $\delta$, while in Fig. 2, it remains constant for $\delta \approx 10^{-6}$. Hence, in contrast to both figures, the choice of the smaller value of the model parameter $\delta$ is preferred. The rotational velocity is unaffected for different smaller values 
of the model parameter $\delta$. Thus, it is interesting to explore the galactic dynamics for the smaller values of $\delta$.

\section{Galactic dynamics via effective $f(R)$ rotational velocity}

Before exploring the galactic dynamics, it is important to make a comment on the formula developed in Eq. (28). This formula will only be used for the acceleration due to point sources. A general development of the rotational velocity formula requires its integration with the disk density profile such that the system is treated as an extended source [55-57]. We will not attempt to solve such an integrated system here and plan to do this by including the Tully-Fisher relation in our future work.

Here, we discuss the simple formula in $f(R)$ spacetime background by treating the gravitating system (galaxies) as a point source. Hence, Eq. (28) suggests that we can fit only the velocities of test stars and $H_{\alpha}$ and $\mathrm{HI}$ profiles that rotate quite far from the disk of the matter of the galaxy. The flatness profile of the galaxy rotation curves is actually associated with this region. Therefore, we trace the test mass in such region according to Eq. (28). For instance, if we assume our galaxy to be a point source and trace the test mass profile beyond its typical disk size $(\approx 15 \mathrm{kpc})$ in $f(R)$ spacetime background with the estimated mass, $M \approx(7 \pm 2.5) \times 10^{11} M_{\odot}$, then we get the observed velocity fit $[60,61]$ for the model parameter $\delta \approx 10^{-6}$ on scaling the $f(R)$ background galactic free parameter $\left(r_{0}\right)$. On the basis of the plot of our Milky Way obtained for the $f(R)$ rotational velocity as in Fig. 3, we argue that beyond the typical galactic size, the rotational curve exhibits a nearly constant velocity profile, which is a nice feature of our theoretical model. This nature arises only because of the $f(R)$ spacetime background potential. There is an initial rise observed in the rotational velocity beyond the visible boundaries of the typical galaxy. Such a bump is also observed in the THINGS (The HI Nearby Galaxy Survey) rotation curve at about $15 \mathrm{kpc}$, which is considered to be the source of information of the test mass motion along the two stretched spiral arms [62]. Since we do not consider any dark matter profile, it may be due to the fact that beyond the disk of galaxy matter, the geometric effect of the $f(R)$ background becomes significant for the suitable values of the model parameter $\delta$ and the galactic scaling parameter $r_{0}$ and hence we get the expected result. Generally, the outer rotation curves are not perfectly flat in the sense that the velocity gradient, i.e., $\frac{\mathrm{d} V}{\mathrm{~d} r}$, is zero. But there is some specific range of radii over which the galactic velocity profile is nearly constant. However, for those cases where extended galactic data is available, the flattening profile of the rotation curves has always been noticed [62].

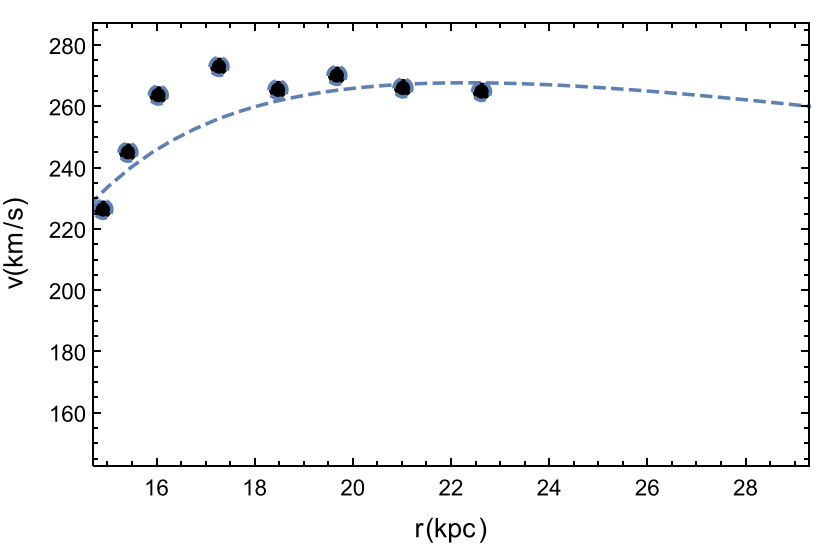

Fig. 3 Theoretical galactic rotation curve external to the typical visible end of the Milky Way galaxy. The black dots show the observed galactic rotation curve in the outer region of galactic luminous disk of matter [60,61]. The dashed curve is the theoretical rotation curve in $f(R)$ background beyond the galactic disk matter demonstrating that the galaxy can be treated as a point source having the typical mass $(7 \pm 2.5) \times 10^{11} M_{\odot}$ and galactic size (about $\left.15 \mathrm{kpc}\right)$. The plot is obtained for $\delta \approx 10^{-6}$ with the galactic scale parameter $r_{0}=10^{2.623}$ $\mathrm{kpc} \approx 419.280 \mathrm{kpc}$

Now, for exploring the actual trace of the test mass according to Eq. (28), we prefer to use those galaxies for which the rotation velocity data point is available far from the disk of galaxy matter. For instance, we take the data for NGC 3198 from $[63,64]$ and plot the modified rotational velocity curve versus distance. In Fig. 4, the outer profile of the rotation curve for NGC 3198 is shown.

Thus, as an important diagnostic test of the proposed model for the clustered dark matter at the local galactic scales, we find a nice agreement for the rotational velocity profile obtained with the modified rotation velocity formula in an extended manner, which matches with the observed behaviour of the rotational velocity curve far from the galactic disk of matter $[60,61,63,64]$.

\section{Summary and conclusion}

We have studied the power-law $f(R)$ gravity model for the explanation of the clustered galactic dark matter problem proposing the cosmological background geometry. We have discussed the suitable spacetime geometry and the line element in the weak field limit for the bounded system for obtaining the integrated effective acceleration profile of the test mass in $f(R)$ cosmological background geometry along with its Newtonian profile and furthermore we calculated the effective $f(R)$ rotational velocity. The variation of the effective $f(R)$ rotational velocity with the model parameter is discussed in Figs. 1 and 2. We have explored the rotational velocity expression for the point sources only and would attempt to generalize it for the extended system by integrat- 


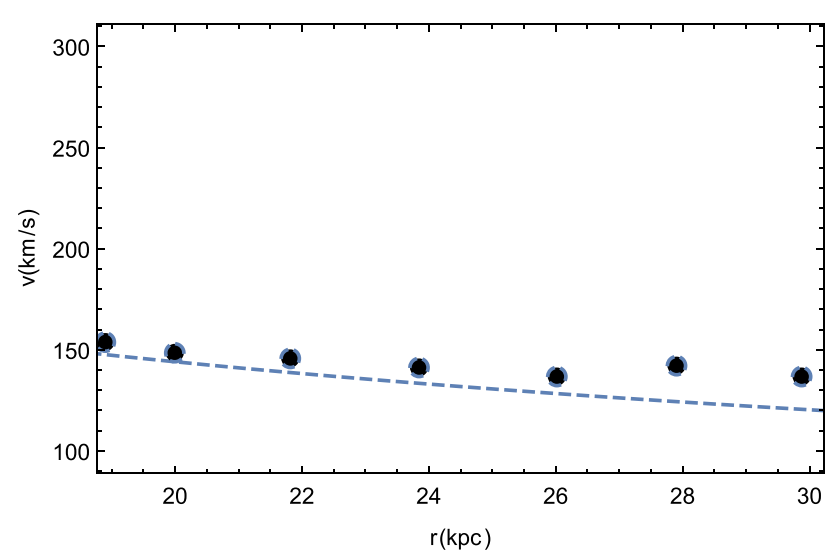

Fig. 4 The rotation curve external to the typical galactic disk of matter for the galaxy NGC 3198. The dots show the observed [63,64] behaviour in the outer region of galactic luminous disk of matter. The dashed line shows the theoretically predicted rotation curve far from the galactic disk matter with $M \approx 11.0 \times 10^{10} M_{\odot}$. The plot is obtained with $\delta \approx 10^{-6}$ and the galactic scale parameter $r_{0}=10^{2.250} \mathrm{kpc} \approx 177.80$ $\mathrm{kpc}$

ing it with the disk density profile and also by including the Tully-Fisher relation in our future work. Therefore, the calculated formula for the $f(R)$ rotational velocity is suitable for the discussion of those galaxies for which the data points (corresponding to the flat rotational velocity profile) were available far from the disk of galaxy matter as the flatness problem is primarily associated with such regions. The possible fits of the $f(R)$ rotational velocity profile are discussed in Figs. 3 and 4 via the free galactic scaling parameter for a particular system. Such an emergence of an extra scaling length is a characteristic feature of any power-law $f(R)$ gravity model, which is clearly discussed in [39-41]. This extra scaling length in our case may be the size of the spherical galactic halo of scalaron clouds. If this is so, then a further constraint can be imposed on $\delta$ via the lensing angle also. However, we find the constraint on the $f(R)$ model parameter $(\delta)$ via the rotational velocity profile of typical massive gravitating systems (galaxies) to be $O\left(10^{-6}\right)$.

Our result closely matches with the result of Clifton's work [1,2], where the constraint on $\delta$ is identified within the PPN (Parameterized-Post Newtonian) formalism for the same model via discussing the effects of expansion on the equation of motion for a test mass with the help of suitable spacetime metric; the constraints on $\delta$ from the Cassini Space probe are obtained: $\delta=-1.1 \pm 1.2 \times 10^{-5}$. Also, our result is generally different from the result of Capozziello et al. and other authors because we work with point sources (constant mass) only [55-57]. They reported a large deviation in the exponent of the power-law $f(R)$ gravity (which ranges from 1.2 to 2.5 ) to explain the observed behaviour of the galactic rotation curves for different luminosity profiles of galaxies.
Hence, for a point-like source in $f(R)$ cosmological background geometry, we have extended galactic rotational velocity profiles, sufficient to tackle the flatness issue.

Acknowledgements The authors thank IUCAA, Pune, for the facilities during which a part of the present work was completed under the associateship programme. VKS also thanks Varun Sahni and S. S. Mishra for their constant support and motivation.

Data Availability Statement This manuscript has no associated data or the data will not be deposited. [Authors' comment: This manuscript has no associated data to be deposited.]

Open Access This article is licensed under a Creative Commons Attribution 4.0 International License, which permits use, sharing, adaptation, distribution and reproduction in any medium or format, as long as you give appropriate credit to the original author(s) and the source, provide a link to the Creative Commons licence, and indicate if changes were made. The images or other third party material in this article are included in the article's Creative Commons licence, unless indicated otherwise in a credit line to the material. If material is not included in the article's Creative Commons licence and your intended use is not permitted by statutory regulation or exceeds the permitted use, you will need to obtain permission directly from the copyright holder. To view a copy of this licence, visit http://creativecomm ons.org/licenses/by/4.0/.

Funded by SCOAP 3 .

\section{References}

1. T. Clifton, Class. Quantum Gravity 23, 7445 (2006)

2. T. Clifton, J.D. Barrow, Phys. Rev. D 72, 103005 (2005)

3. V.C. Rubin, W.K. Ford, N. Thonnard, Astrophys. J. 238, 471 (1980)

4. V.C. Rubin, D. Burstein, W.K. Ford, N. Thonnard, Astrophys. J. 289, 81 (1985)

5. A. Borriello, P. Salucci, Mon. Not. R. Astron. Soc. 323, 285 (2001)

6. R.H. Sanders, The Dark Matter Problem: A Historical Perspective (Cambridge University Press, Cambridge, 2010)

7. D. Clowe, M. Bradac et al., Astrophys. J. 604, 596 (2004)

8. D. Clowe, M. Bradac et al., Astrophys. J. Lett. 648, L109 (2006)

9. A.G. Riess et al. (Supernova Search Team Collaboration), Astron. J. 116, 1009 (1998)

10. S. Perlmutter et al., Astrophys. J. 517, 565 (1999)

11. D.N. Spergel et al., Astrophys. J. Suppl. 148, 97 (2003)

12. N. Aghanim, Y. Akrami, et al., Planck 2018 results (2018). arXiv: $1807.06209 \mathrm{v} 2$

13. S. Capozziello, M. De Laurentis, Phys. Rep. 509, 167 (2011)

14. A.D. Felice, S. Tsuzikawa, Living Rev. Relativ. 13, 3 (2010)

15. Y. Fujii, K. Maeda, The Scalar-Tensor Theory of Gravitation (Cambridge University Press, Cambridge, 2003)

16. V. Faraoni, Cosmology in Scalar-Tensor Gravity (Springer, Berlin, 2004)

17. J.D. Bekenstein, Phys. Rev. D 70, 083509 (2004)

18. J.W. Moffat, JCAP 0603, 004 (2006)

19. A. Belenchia et al., Rep. Prog. Phys. 81, 036001 (2018)

20. L. Amendola, Dark Energy: Theory and Observations (Cambridge University Press, Cambridge, 2010)

21. M. Milgrom, Astrophys. J. 270, 365 (1983)

22. J.R. Brownstein, J.W. Moffat, Astrophys. J. 636, 721 (2006)

23. R.H. Sanders, E. Noordermeer, Mon. Not. R. Astron. Soc. 379, 702 (2007)

24. H.A. Buchdahl, Mon. Not. R. Astron. Soc. 150, 1 (1970)

25. A.A. Starobinsky, Phys. Lett. B 91, 99 (1980) 
26. A.A. Starobinsky, JETP Lett. 86, 157 (2007)

27. H. Motohashi, A.A. Starobinsky, J. Yokoyama, Int. J. Mod. Phys. D 20(8), 1347 (2011)

28. J.A.R. Cembranos, Phys. Rev. Lett. 102(14), 141301 (2009)

29. G.J. Olmo, Phys. Rev. Lett. 95(26), 261102 (2005)

30. S. Capozziello, M. De Laurentis, G. Lambiase, Phys. Lett. B 715, $1(2012)$

31. V. Faraoni, S. Capozziello, Beyond Einstein Gravity: A Survey of Gravitational Theories for Cosmology and Astrophysics, vol. 170 (Springer, Dordrecht, 2011)

32. V. Sahni, The Physics of the Early Universe. Lecture Notes in Physics, vol. 653 (Springer, Berlin, 2004)

33. V. Sahni, A. Starobinsky, Int. J. Mod. Phys. D 15, 2105 (2006)

34. W. Hu, I. Sawicki, Phys. Rev. D 76, 064004 (2007)

35. S.A. Appleby, R.A. Battye, Phys. Lett. B 654, 7 (2007)

36. T.P. Waterhouse (2006). arXiv:astro-ph/0611816

37. T.P. Sotiriou, V. Faraoni, Rev. Mod. Phys. 82, 451 (2010)

38. C. Burrage, J. Sakstei, Living Rev. Relativ. 21, 1 (2018)

39. S. Capozziello et al., JCAP 06, 044 (2017)

40. S. Capozziello, A. Stabile, A. Troisi, Class. Quantum Gravity 24, 2153 (2007)

41. S. Nojiri, S.D. Odintsov, Phys. Rep. 505, 59 (2011)

42. V. Sahni, L. Wang, Phys. Rev. D 62, 103517 (2000)

43. S.S. Mishra, V. Sahni, Y. Shtanov, JCAP 06, 045 (2017)

44. B.K. Yadav, M.M. Verma, JCAP 10, 052 (2019)

45. V. Sahni, Y. Shtanov, A. Toporensky, Class. Quantum Gravity 32, 182001 (2015)

46. A.E. Allahverdyan, V.G. Gurzadyan, Phys. Rev. E 93, 052125 (2016)
47. B.K. Yadav, M.M. Verma, Int. J. Mod. Phys. D 26, 1750183 (2017)

48. B.K. Yadav, M.M. Verma, Int. J. Mod. Phys. D 27, 1850002 (2018)

49. R.H. Dicke, P.J.E. Peebles, Phys. Rev. Lett. 12, 435 (1964)

50. J.L. Anderson, Phys. Rev. Lett. 75, 3602 (1995)

51. Jr. G.A. Baker (2001). arXiv:astro-ph/0112320v1

52. F.I. Cooperstock, V. Faraoni, D.N. Vollick, Astrophys. J. 503, 61 (1998)

53. G.C. McVittie, Mon. Not. R. Astron. Soc. 93, 325 (1933)

54. S. Nesseris, L. Perivolaropoulos, Phys. Rev. D 70, 123529 (2004)

55. S. Capozziello, V.F. Cardone, A. Troisi, Mon. Not. R. Astron. Soc. 375, 1423 (2007)

56. S. Capozziello, V.F. Cardone, A. Troisi, JCAP 0608, 001 (2006)

57. C.F. Martins, P. Salucci, Mon. Not. R. Astron. Soc. 381, 1103 (2007)

58. T. Padmanabhan, Gravitation: Foundations and Frontiers (Cambridge University Press, Cambridge, 2010)

59. J.A. Peacock, Cosmological Physics (Cambridge University Press, Cambridge, 1999)

60. M. Pato, F. Iocco, SoftwareX 6, 54 (2017)

61. A. Klypin, H. Zhao, R.S. Somerville, Astrophys. J. 573, 597 (2002)

62. W.J.G. De Blok et al., Astrophys. J. 136, 2648 (2008)

63. E.V. Karvkes, P. Salucci, G. Gentile, Astron. Astrophys. 578, 13 (2015)

64. K.G. Begeman, Astron. Astrophys. 223, 47 (1989) 